\title{
WASHINGTON UNIVERSITY
}

Department of Applied Mathematics and Computer science School of Engineering and Applied Science

\author{
MINIMIZING COSTS INVOLVING \\ ECONOMIES OF SCALE:
}

A GLOBAL OPTIMIZATION PROBLEM

by

Philip B. Zwart

This research was supported in part by the Atomic Energy Commission under Research Contract No. A(11-1)-1493 and by the Department of Defense under Themis Grant No. F44620-69-C-0116.

Report No. COO-1493-33

(CSSE 704)

TES report was prepared as an account of Governmeat apponsored work. Nelther the Unitod Btates, nor the Commisoton, nor any person acting on behalt of the Comoulasion:

A. Makes any warranty or represcatstion, expressed or Implled, whth respect to the arcuracy, completeness, or usefunese of the tifformation contenined in this report, or that the use of any linlormation, apparatus, method, or proceses disclosed in thes report many not lafringo

privately owned rights; or
B. Assumes any liablitites with reapect to the use of, or for danngea resulung frost the

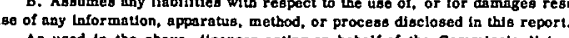

As used in the above. "person acturg on bechalf of the commiselon" Includes any cm-" ployes or contractor of the commiselon, or employee of such contrector. to the extent that Buch employee or contractor of the Commileston, or employee of such contractor prepares, disseminates, or provides access to, any informatlon puranant to bls employment or contrac 


\section{DISCLAIMER}

This report was prepared as an account of work sponsored by an agency of the United States Government. Neither the United States Government nor any agency Thereof, nor any of their employees, makes any warranty, express or implied, or assumes any legal liability or responsibility for the accuracy, completeness, or usefulness of any information, apparatus, product, or process disclosed, or represents that its use would not infringe privately owned rights. Reference herein to any specific commercial product, process, or service by trade name, trademark, manufacturer, or otherwise does not necessarily constitute or imply its endorsement, recommendation, or favoring by the United States Government or any agency thereof. The views and opinions of authors expressed herein do not necessarily state or reflect those of the United States Government or any agency thereof. 


\section{DISCLAIMER}

Portions of this document may be illegible in electronic image products. Images are produced from the best available original document. 


\section{ABSTRACT}

Cost minimization with an objective function which reflects the effects of economies of scale can lead to nonlinear programming problems which possess more than one local optimum. A computational procedure is presented for such cost minimization subject to linear inequality constraints. 
I. Introduction:

The unit cost of purchased items frequently decreases with increased order quantities. Figure 1 shows how the total cost might depend on quantity ordered, when the sellers pricing policy is:

$C_{1}$ per unit for the first $P_{1}$ units,

$C_{2}$ per unit for the next $P_{2}$ units,

$C_{3}$ per unit. for the next $P_{3}$ uni,ts,

$C_{4}$ per unit for the rest of the order.

In a similar fashion unit production costs tend to decrease with amount produced. Figure 2 shows how total cost might depend on quantity produced in a situation where the employees' familiarity. with the job brings: about greater productivity rates for larger production quantities.

Iinear programing is concerned with finding the combination of order (or production) quantities, which minimize a linear cost function subject to linear constraints. A linear cost function, $\sum_{i=1}^{n} c_{i} x_{i}$, represents situations where unit costs are independent of order quantity. An objective function which reflects economies of scale must be nonlinear.

In this paper, we: are concerned with minimizing, subject to linnear inequalities, a cost function of the form $f(x)=\sum_{i=1}^{n} f_{i}\left(x_{i}\right)$, where each $f_{i}$ reflects economics of scale, such as occur in figures 1 and 2. Such an objective function is concave. $f(x)$. 


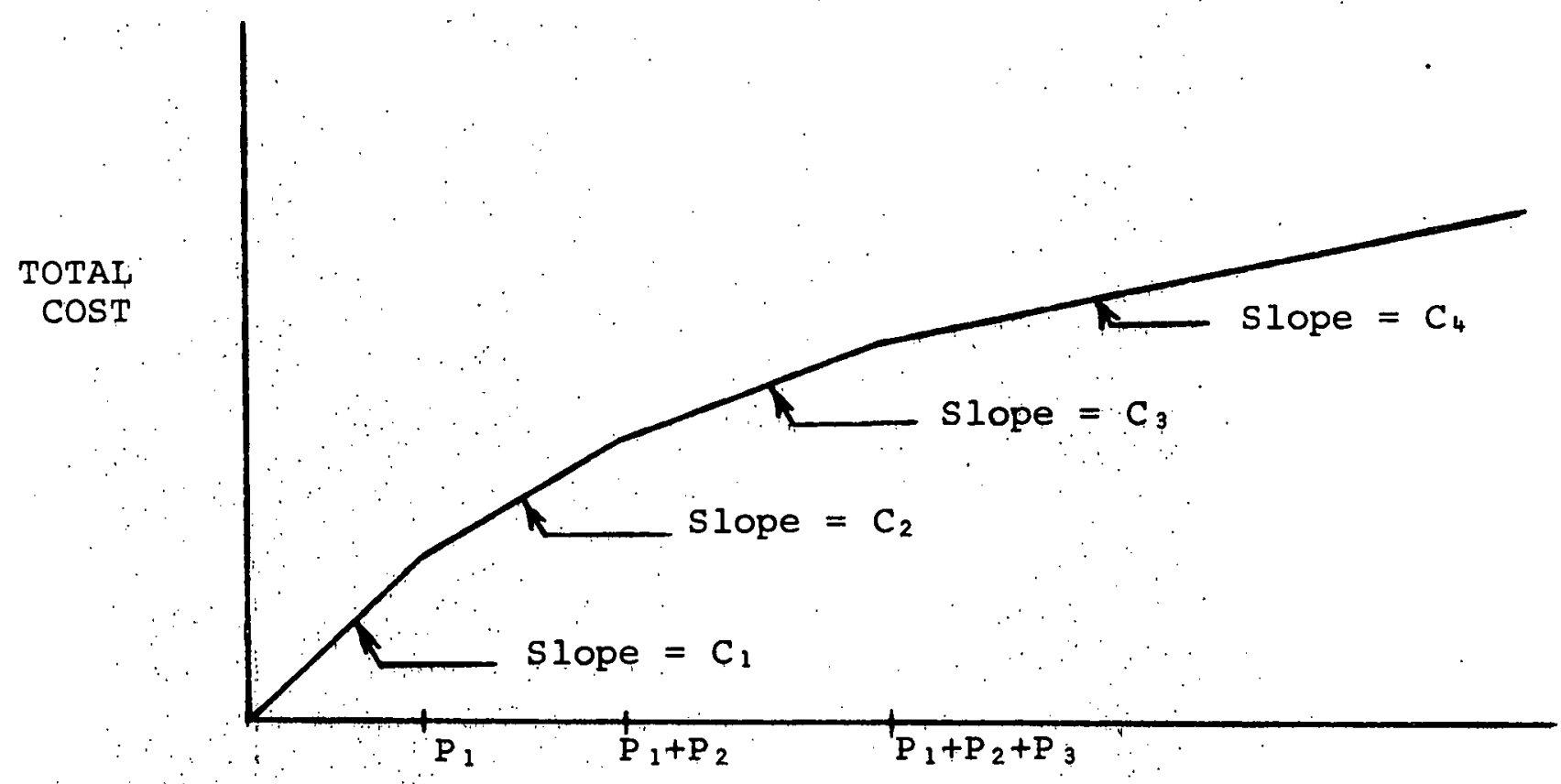

No: of Units Ordered

Figure 1

Order Costs

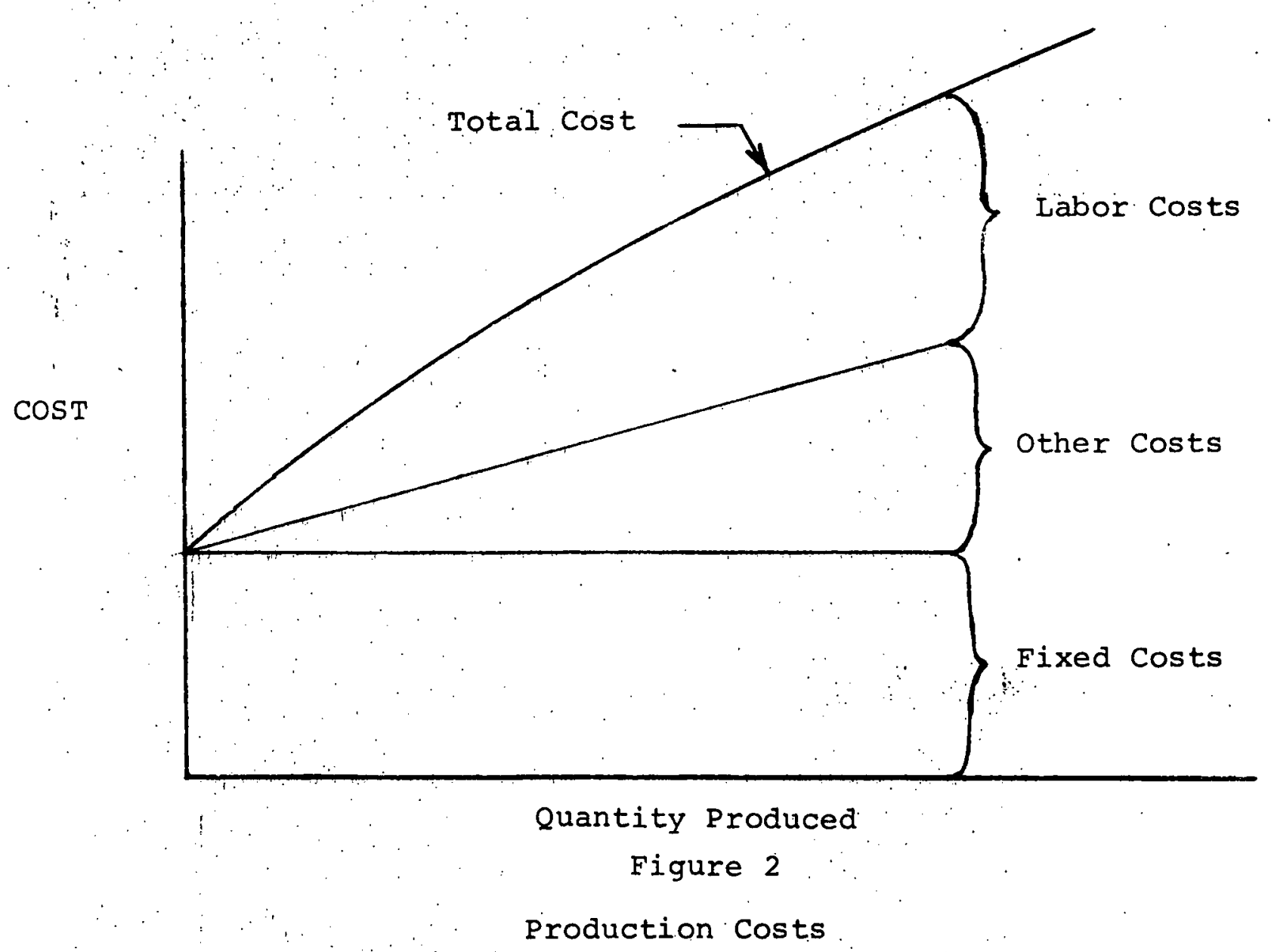


restricted to a convex set may possess many local optima. Not only is linear programming unsuitable, but also nonlinear programming techniques, such as proposed by Griffith and stewart [1], Rosen [5]; Goldfarb [2], etc., are unsuitable because they merely seek out a local optimum.

Some work has been done on minimizing a concave function subject to linear inequality constraints, Tui [6] presents a couple of approaches to this problem. Ritter [3], [4], deals with the case where the $f_{i}$ 's are any quadratic functions. It has been shown in [7] that the first method presented by Tui, and the procedure of Ritter's are not computationally sound. The approach presented in this paper, is much like Tui's second suggestion, but takes particular advantage of special properties of a concave cost function which reflects economies of scale. The problem is defined in section II. Then special aspects of the problem are discussed in section II.I. The algorithm is presented in section IV. It is not known whether or not the procedure is finite. So a method for obtaining a lower bound on the global minimum is described in the last section. 
II. The Problem:

$$
\begin{aligned}
& \text { Our problem is: } \\
& \text { Maximize. } f(x)=\sum_{i=1}^{n} f_{i}\left(x_{i}\right) \\
& \text { Subject to: } \quad A x \leq b \\
& x \geq 0 \text {. }
\end{aligned}
$$

where $\mathrm{x}$ is an $\mathrm{n}$-component column vector, $\mathrm{b}$ is a given m-component column vector, $A$ is a given $m \times n$ matrix, and $s^{\ell}$ and $\mathrm{S}^{\mathrm{u}}$ are given $\mathrm{n}$-component column vectors, and the $\mathrm{f}_{i}$ are nonnegative continuous piecewise differentiable functions ' whose derivatives are monotonically non-increasing and satisfy $0 \leq \mathrm{s}^{\ell} \leq \frac{\mathrm{df}}{\mathrm{dx}} \leq \mathrm{s}^{\mathrm{u}}$.

The requirements on the $f_{i}$ are consistent with our desire that they represent total cost functions which reflect economies of scale. Concavity follows from the fact that unit costs. $\left(\frac{d f}{d x}\right)$ do not increase as quantity increases. The requirement that $0 \leq s^{l} \leq \frac{d f}{d x} \leq s^{u}$, merely says that each item's cost function $f_{i}$, possesses some maximum unit cost $s_{i}^{u}$ (which is $\left.\frac{d f}{d x_{i}}\right|_{x_{i}=0}$ ). and some minimum unit cost $s_{i}^{\ell}$ (which is $\lim _{x_{i} \rightarrow \infty} \frac{d f_{i}}{d x_{i}}$ ). 
III. Some Special Considerations:

A few ideas related to our particular problem should be presented prior to a description of the algorithm.

a. Extension of the Objective Function.

The $f_{i}$ 's, being cost functions, are not defined for negative $x_{i}$ 's, which are excluded by the requirement $x \geq 0$ in (1). The $f_{i}^{\prime} s$ can be extended to include negative $x_{i}$ 's by using:

$$
\tilde{f}_{i}\left(x_{i}\right)=\left\{\begin{array}{l}
f_{i}(0)+s_{i}^{u} x_{i}, x_{i}<0 \\
f_{i}\left(x_{i}\right), x_{i} \geq 0 .
\end{array}\right.
$$

Claim: $\tilde{f}_{i}$ is concave.

Proof: $\frac{d \tilde{f}_{i}}{d x_{i}}$ is a monotonically nonincreasing function of $x_{i}$. Therefore, $\tilde{f}_{i}$ is concave.

b. An Additional Constraint.

Let $v$ represent an objective function value. Set

$$
I(v)=\left\{i \mid \exists Y_{i}(v)>03 f_{i}\left(y_{i}(v)\right)=v\right\}
$$

Note: If $s_{j}^{l}=0$, then it is possible that. $j \notin I(v)$.

Claim: $\quad x \geq 0, \sum_{i \in I(v)}\left(x_{i} / y_{i}(v)\right)>1$ implies $f(x)>v$ :

Proof: Suppose $x$ satisfies $\sum_{i \varepsilon I(v)}\left(x_{i} / y_{i}(v)\right)=\alpha>I$. 
III. Some Special Considerations:

A few ideas related to our particular problem should be presented prior to a description of the algorithm.

a. Extension of the Objective Function.

The $f_{i}$ 's, being cost functions, are not defined for 'negative $x_{i}$ 's, which are excluded by the requirement $x \geq 0$ in (1). The $f_{i}^{\prime}$ 's can be extended to include negative $x_{i}^{\prime}$ ' by using:

$$
\tilde{f}_{i}\left(x_{i}\right)=\left\{\begin{array}{l}
f_{i}(0)+s_{i}^{u} x_{i}, x_{i}<0 \\
f_{i}\left(x_{i}\right), x_{i} \geq 0 .
\end{array}\right.
$$

Claim: $\tilde{f}_{i}$ is concave.

Proof: $\frac{d \tilde{f}_{i}}{d x_{i}}$ is a monotonically nonincreasing function of $x_{i}$. Therefore, $\tilde{f}_{i}$ is concave.

b. An Additional Constraint.

Li:t v represent an cbjective function value. Set

$$
I(v)=\left\{i \mid \exists z_{i}(v)>03 f_{i}\left(y_{i}(v)\right)=v\right\}
$$

Note: If $s_{j}^{l}=0$, then it is possible that $j \notin I(v)$.

Claim: $\quad x \geq 0, \sum_{i \in I(v)}\left(x_{i} / y_{i}(v)\right)>1$ implies $f(x)>v$ :

Proof: Suppose $x$ satisfies $\sum_{i \in I(v)}\left(x_{i} / y_{i}(v)\right)=\alpha>1$ 
Then, set $z_{i}=\left\{\begin{array}{l}0, \text { if } i \notin I(v) \\ x_{i}, \text { if } i \in I(v)\end{array}\right.$

We have $\sum_{i \varepsilon I(v)}\left(z_{i} / \alpha y_{i}(v)\right)=1$ and

$$
z=\sum_{i \in I(v)}\left(z_{i} / \alpha y_{i}(v)\right) \alpha y_{i}(v) e^{i} \text {, where } e^{i} \text { are the coordinate vectors, }
$$

i.e. $\quad e_{j}^{i}=\delta_{i j}$.

Thus, $f(z) \geq \sum_{i \in I(v)}\left(z_{i} / \alpha y_{i}(v)\right) f_{i}\left(\alpha y_{i}(v)\right)$

$>\sum_{i \in I(v)}\left(z_{i} / \alpha y_{i}(v)\right) v$

$$
\geq v
$$

And, $f(x)=\sum_{i=1}^{n} f_{i}\left(x_{i}\right) \geq \sum_{i \in I(v)} f_{i}\left(x_{i}\right)=f(z)>v \cdot a(v)$ will

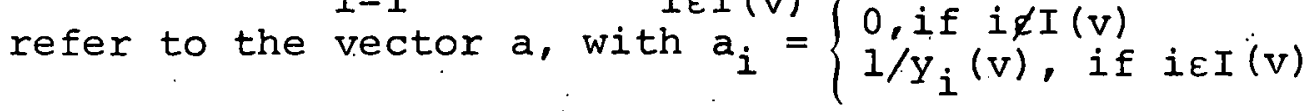

c. One-Dimensional Search Result Recognition.

Suppose we wish to find $\min _{t \rightarrow 0} \tilde{f}(y+t d)$. $n$ where $y$ and $d$ are fixed, and $\left.\frac{d \tilde{f}}{d t}(y+t d)\right|_{t=0} ^{>0} \cdot \sec ^{t>0} s(d)=\sum_{i=1}^{n} \min \left(s_{i}^{\ell} d_{i}, s_{i}^{u} d_{i}\right)$.

Claim: $\min _{t \geq 0} \tilde{f}(y+t d)=\tilde{f}(y) \quad$ iff $s(a) \geq 0$ 
Proof: $\tilde{f}(y+t d)$ is a concave function of $t$.

$$
\begin{aligned}
& \begin{aligned}
& \tilde{f}(y)<\tilde{f}(y+t d) \text { iff } \frac{d \tilde{f}}{d t}(y+t d)>0, t>0 . \\
& \text { That is, iff } \lim _{t \rightarrow \infty} \frac{\tilde{d}}{d t}(y+t d)>0 . \\
& \text { Now } \lim _{t \rightarrow \infty} \frac{d \tilde{f}}{d t}(y+t d)=\sum_{i=1}^{n} \lim _{t \rightarrow \infty} \frac{d \tilde{f}}{d t}\left(y_{i}+t d_{i}\right) \\
&=\sum_{i=1}^{n} \min \left(s_{i}^{\ell} d_{i}, s_{i}^{u} d_{i}\right) .
\end{aligned}
\end{aligned}
$$

d. Constraint Addition After One-Dimensional Searches.

Suppose $z, \theta^{1} ; \ldots, \theta^{\mathrm{n}}$ have been determined such that the $\theta^{j}, j=1, \ldots, n$ are linearly independent and

$$
\begin{aligned}
& \tilde{f}(z) \geq v_{r} \\
& \tilde{f}\left(z+t_{j} \theta^{j}\right)=v_{r} t_{j}>0, j \in J \\
& \tilde{f}\left(z+t \theta^{i}\right) \geq v_{r} \text { for all } t \geq 0, i \not J .
\end{aligned}
$$

Let $a$ be the uniquely determined $n$ vector which satisfies

$$
\begin{aligned}
& a \cdot t_{j} \theta^{j}=1, \quad j \in J \\
& a \cdot \theta^{i}=0, \quad i \notin J .
\end{aligned}
$$

Claim: If $x=z+\sum_{j=1}^{n} \alpha_{j} \theta^{j}, \theta_{j \geq 0}$, satisfies $a \cdot x<1+a \cdot z$. then $\tilde{\mathrm{f}}(\mathrm{x}) \geq \mathrm{v}$. 
Proof: $a \cdot x=a \cdot z+\sum_{j=1}^{n}\left(a_{j} / t_{j}\right) a \cdot t_{j} \theta^{j}$

$$
=a \cdot z+\sum_{j \in J}\left(\alpha_{j} / t_{j}\right)
$$

$$
a \cdot x<1+a \cdot z \quad \sum_{j \in J}\left(\alpha_{j} / t_{j}\right)<1
$$

Now, $x=z+\sum_{j=1}^{n} \alpha_{j} \theta^{j}$

$$
\begin{gathered}
=z\left(1-\sum_{j \in J}\left(\alpha_{j} / t_{j}\right)\right)+\sum_{j \in J}\left(\alpha_{j} / t_{j}\right)\left(z+t_{j} \theta^{j}\right)+\sum_{j \not J J} \alpha_{j} \theta^{j} \\
=\delta z+\sum_{j \in J}\left(\alpha_{j} / t_{j}\right)\left(z+t_{j} \theta^{j}\right)+\sum_{j \notin J} \delta\left(z+\left(\alpha_{j} / \delta\right) \cdot \theta^{j}\right),
\end{gathered}
$$

where $\delta=\left(1-\sum_{j \in J}\left(\alpha_{j} / t_{j}\right)\right) /(1+n-$ no. of subscripts in J). And, since $\tilde{f}$ is concave,

$$
\begin{aligned}
& \tilde{f}(x) \geq \delta \tilde{f}(z)+\sum_{j \in J}\left(\alpha_{j} / t_{j}\right) \tilde{f}\left(z+t_{j} \theta^{j}\right)+\sum_{i \neq J} \delta \tilde{f}\left(z+\left(\alpha_{j} / \delta\right) \theta^{j}\right)
\end{aligned}
$$

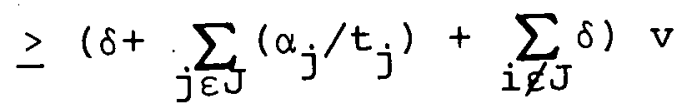

$$
\begin{aligned}
& \text { = v. : }
\end{aligned}
$$

The uniquely determined a will be referred to as a $(z, \theta)$. 
e. Finding a Local Minimum Extreme Point.

Consider the following procedure:

i) Let $z$ be an extreme point for $A^{*} \leq b^{*}, x \geq 0$, which does not minimize $\nabla f(z) \cdot x$.

Find $\hat{z}$, an extreme point of $A^{*} x \leq b^{*}, x \geq 0$, which minimizes $\nabla f(z) \cdot x$.

ii) If $\hat{z}$ also minimizes $\nabla f(\hat{z}) \cdot x$, then $\hat{z}$ is a local minimum extreme point. If not, then use $\hat{z}$ as a new $z$ and go to i).

claim: The above procedure yields a local minimum extreme point in a finite number of steps.

Proof: Since $\nabla f(z) \cdot(\hat{z}-z)<0$, and $f$ is concave, we must have $f(\hat{z})<f(z)$. The procedure yields a sequence of distinct. (different values of $f$ ) extreme points. Since there are only a finite number of extreme points, the procedure must terminate.

IV. The Algorithm:

We start with $A^{*}=A, b^{*}=b$. And we assume that the original constraint set possesses a feasible solution.

1. Find an extreme point, $z$, which is a local minimum for $f$ subject to $A^{*} x \leq b^{*}$. If there are no feasible solutions, then $f$ takes on its global minimum at $x^{*}$. Otherwise, go to step $2 . a$.

2.a. From those constraints which hold as equalities at $z$; pick $n$ constraints; say $a_{i_{j}} \cdot x \leq b_{i_{j}}, j=1, \ldots, n$, which 
are linearly independent, such that $\mathbf{z}$ is a local minimum of $f$ over $\left\{x \mid a_{i j} \cdot x \leq b{ }_{i j}, j=1, \ldots, n\right\}$. Go to step $2 . b$.

b. Determine $n$ vectors $\theta^{1}, \ldots, \theta^{n}$ as solutions to $a_{i_{j}} \cdot \theta^{j}=-\delta_{i_{j} j}$ (Kronecker delta) $j=1, \ldots, n$. Go to step 2.c. c. Set $J=\left\{j ! a t t_{j}>0\right.$, such that $\left.\tilde{f}\left(z+t_{j} \theta^{j}\right)=f\left(x^{*}\right)\right\}$. (See section III b.). Go to step $2 . d$.

d. If, for some $j \varepsilon J, \exists t>0$, such that $z+t \theta^{j}$. satisfies $A^{*} x \leq b *$, and $f\left(z+t \theta^{j}\right)<f\left(x^{*}\right)$ then go to step 2e. Otherwise go to 3 .

e. Determine $\tilde{t}_{j}$. jहJ, as

$$
\tilde{t}_{j}=\max \left\{t \mid z+t \theta^{j} \text { satisfies } A^{*} x \leq b *\right\} \text {. }
$$

Set $x^{*}=t>0, z+t_{\hat{j}} \theta^{\hat{j}}$ where $\hat{j}$ is determined by $f\left(z+\tilde{t}_{\hat{j}} \theta^{\hat{j}}\right)=\underset{j \in J}{\max }\left\{f\left(z+\tilde{t}_{j} \theta^{j}\right\}\right.$. Go to step $2 \mathrm{c}$.

3. Add $-a(z, \theta) \cdot x \leq-a(z, \theta) \cdot z-1$ (see section III d.) to the constraint set $A^{*} \mathrm{x} \leq \mathrm{b}^{*}$. Go to step 4 .

4. Add $a\left(f\left(x^{*}\right)\right) \cdot x \leq 1$ to the constraint set $A^{*} x \leq b *$ (see section III b.). Go to step 1. (Notice that if this has been done before, then the new constraint can replace the previously added constraint.)

To see that termination in step 1 implies that $x^{*}$ gives the global minimum of $f$, we must show that the constraints added 
in 3 and 4 eliminate only feasible points $y$ such that $f(y)>$ global minimum of $f$.

In section III b., it is shown that all points $y$ eliminated by $a\left(f\left(x^{*}\right)\right) \cdot x<1$ must satisfy $f(y)>f\left(x^{*}\right)$. And, since $f\left(x^{*}\right) \geq$ global minimum of $f$, step 3 is $0 . k$.

In section III $d$. , it is shown that all points of $y=\sum_{i=1}^{n} \alpha, \theta^{i}, \alpha_{i} \geq 0$, which satisfy $a(z, \theta) \cdot y<1+a(z, \theta) \cdot z$ must satisfy $\tilde{f}(y) \geq f\left(x^{*}\right)$. Now any point which satisfies $a_{i j} \cdot \underline{x}_{i_{j}}, j=1, \ldots, n$ can be expressed as $\sum_{i=1}^{n} \alpha_{i} \theta^{i}, \alpha_{i} \geq 0$, where the $\theta^{i}$ are determined by step $2 b$. Thus, in step 4 , any

feasible point $y$, eliminated by the new constraint, must have $\tilde{f}(y)>f\left(x^{*}\right) \geq g$ lobal minimum of $f$.

It is an open question as to whether or not the procedure is finite. However, it should be noted that in step $1:, z$ could be chosen as any non-degenerate extreme point. In fact, if a particular local minimum, yields little reduction of the feasible region, it may be better to choose some other extreme point.

The next section describes how to obtain a bound on the global minimum of $f$, if it is necessary to discontinue calculations before termination by step 1 .

V. Bound on Minimum:

Suppose $z$ is a point of $A x \leq b, x \geq 0$, which is the minimum for some linear function $c \cdot x$, where $c>0$. Claim: Minimum of $f$ subject to $A x \leq b$ is

$$
\geq \min _{j}\left\{f_{j}\left(c \cdot z / c_{j}\right)\right\} \text {. }
$$


Proof: For any feasible $x$, let $y^{k}, k=1, \ldots, n$ represent the $n$ vectors with components

$$
y_{i}^{k}=\delta_{i k} c \cdot x / c_{i} ; k, i=1, \ldots, n .
$$

Then $x_{i}=\left(c_{i} x_{i} / c \cdot x\right) y_{i}^{i}$, and

$$
x=\sum_{k=1}^{n}\left(c_{k} x_{k} / c \cdot x\right) y^{k}
$$

Also, $\sum_{k=1}^{n}\left(c_{k} x_{k} / c \cdot x\right)=1$.

So, $f(x) \geq \sum_{k=1}^{n}\left(c_{k} x_{k} / c \cdot x\right) f\left(y^{k}\right)$.

Now, $f\left(y^{k}\right)=f_{k}\left(c \cdot x / c_{k}\right) \geq f_{k}\left(c \cdot z / c_{k}\right)$.

So, $\left.f(x) \geq \sum_{k=1}^{n}\left(c_{k} x_{k} / c \cdot x\right) f_{k}(c \cdot z) c_{k}\right)$

$$
\begin{aligned}
& \geq \sum_{k=1}^{n}\left(c_{k} y_{k} / c \cdot x\right) \min _{j}\left\{f_{j}\left(c \cdot z / c_{j}\right)\right\} \\
& =\min _{j}\left\{f_{j}\left(c \cdot z / c_{j}\right)\right\} .
\end{aligned}
$$

This result is particularly handy because, if $z$ is $a$ local minimum for $f$ then $z$ is a minimum for $\nabla f(z) \cdot x$. Moreover, if it is known that $x \geq d \geq 0$, then a translation to $\bar{x}=x-d$ shows that $f_{j}\left(c \cdot z / c_{j}\right)$ can be replaced with $f(d)+f_{j}\left(c \cdot z / c_{j}-d_{j}\right)-f_{j}\left(d_{j}\right)$. 


\section{BIBLIOG RAPHY}

[1] Griffith, R.E. and Stewart, R.A., "A Non-Linear Programming Technique for the Optimization of Continuous Processing Systems", Management Science 7 (1961) 379-392.

[2] Goldfarb, D., "Extension of Davidon's Variable Metric Method to Maximization Under Linear Inequality and Equality Constraints", SIAM J. on Applied Math., 17 (1969) 739-764.

[3] Ritter, K., "Stationary Points of Quadratic MaximumProblems", z. Wahrscheinlichkeitstheorie verw. Geb. 4, (1965) 149-i58.

[4] Ritter, K., "A Method for Solving Maximum-Problems with a Nonconcave Quadratic Objective Function", ‥ Wahrscheinlichkeitstheorie verw. Geb. 4, (1966) 340-351.

[5] Rosen, J.B., "The Gradient Projection Method for Non-linear Programming, Part I", SIAM Journal 8, (1960) 181-217.

[6] Tui, Hoanz, "Concave Programming under Linear Constraints", Dokl. Akad. Naul SSR 159 (1964) 32-35; English translation in Soviet Math. Dokl. 5 (1964) 1437-1440.

[7] Zwart, P.B., "Nonlinear Programming: Counterexamples to Global Optimization Algorithms Proposed by Ritter and Tui", recently submitted to $\mathrm{z}$. Wahrscheinlichkeits theorie. 